\title{
A Predictive Study of Wiki Interaction: Can Attitude toward Wiki Predict Wiki Interaction in High-Context Cultures Groups?
}

\author{
Hung-Ling Twu \\ National Chin-Yi University of Technology
}

\begin{abstract}
Wiki technology is more than a technical mechanism to support language learning. It should be seen as an attitude toward social learning technology with consideration for social and cultural factors of the learners. It is not appropriate to assume all students would perceive the social functions of Wikis in the same way and be equally eager to engage in the technology. Learners from different cultural groups may perceive and interact differently in Wiki-based learning environments because the influence of their cultural perceptions regulates their interaction. This study purposes to examine the predictive relationship between attitude toward Wiki and Wiki interaction in a single culture background classroom. It is concluded that there is a medium to strong, positive relationship between attitudes of learners toward language learning in Wiki learning environments and Wiki interaction in a Chinese ESL classroom. Strategies to support and improve attitudes of learners toward language learning in Wiki learning environments are suggested.
\end{abstract}

Keywords: Wiki; ESL, Wiki strategies, interaction; socio-cultural learning

\section{Introduction}

Social and cultural issues, rather than technical issues, are fundamental to social software and networking technologies. Wiki, considered to be a "Web 2.0" technology, is fundamentally grounded in social and cultural aspects to support learning because it facilitates learning through social interaction which includes creating, editing, collaborating, and synthesizing multiple ideas. Educators are consistently seeking creative and imaginative ways to support brainstorming, language learning, creative learning, collaborative learning, and community learning. These cognitive processes rely heavily on social interaction and learners' attitude toward Wiki instruction.

While integrating social networking technologies to support language learning, educators frequently focus on the Wiki's potential learning supremacy in cognitive and mechanical interaction in reading, creating, and editing the Wiki content supplied by the student and Wiki content supplied by others. Wiki is considered to be a "social medium." Social aspects should be accentuated to maximize social interaction in Wiki activities by understanding and examining students' attitudes toward language learning in Wiki environments. 


\section{Literature Review}

\subsection{Wiki as Learning Tools}

The basic concept for using Wiki in an educational setting is in its ability to support effective creation and editing of Web content, capture the cognitive processes involved, and display them as historical context for further cognitive processes. Many argue that the new possibilities of these social networking tools result in a fundamental shift in the way students learn, consume, and construct new artifacts (Braun \& Schmidt, 2006). Their learning would be situated within the domains of creativity and task-based learning. Educators see the potential of engaging learners in Wikibased activities to build online collaborative learning communities and enhance language learning because the values of the activities lie in creating, revising, negotiating, synthesizing, and collaborating multiple contents and communications (Farabaugh, 2007).

Recent studies have concluded that Wiki technologies are effective tools to support indepth learning, higher learning engagements, and knowledge sharing. Matthew and Felvegi (2009) concluded that Wiki learning experiences of students leading to in-depth processing of learning content and Wiki contributions promote better learning outcomes in other classes. To generate better and higher learning engagement in Web 2.0 learning environments requires students to have prior distance learning and technology experiences (Lambert, Kalyuga, \& Capan, 2009) because Web 2.0 learning environments require different learning perceptions and technology skills. Wiki instruction focus on online collaboration; therefore, to engage students in active knowledge sharing and information arranging, students' attitudes toward collaboration must be facilitated (Elgort, Smith, \& Toland, 2009).

\subsection{Wiki and Cultures}

Learners from different cultural and ethnic backgrounds may observe, perceive, interact, and communicate differently due to the social structures of their origins and their cultures. It is inappropriate to assume all students would perceive the social function of Wikis in the same way and be equally eager to engage in the technology. Learners from different cultures, such as the Chinese culture designated as a High-Context Culture (HCC) (Sue \& Sue, 1999), may perceive and interact differently in Wiki-based learning environments because of their cultural perceptions regulating their interaction in social relationships, trust, social interaction, and privacy (Greenhow, Robelia \& Hughes, 2009; Thompson \& Pennycook, 2008). Kessler (2009) found that Mexican students, another HCC group were unwilling to attend to form issues that they were quite capable of correcting through Wiki collaborative writing. These phenomena could happen in Chinese online learning environments. In Chinese culture, students are expected to be humble rather than criticizing other learners. Correction is reserved for the authorities only, like teachers.

\subsection{Wiki and Language Learning}

Researchers have foreseen the potential of integrating Wiki technology to enhance language learning by improving writing skills, collaborative writing, publicizing collaborative writing, and deeper language learning content. Mak \& Coniam (2008) concluded that creativity and task-based learning were fundamentals for authentic writing in ESL for HCC students. Overall, studies concluded that Wiki technologies and instruction enhance the development of writing skills (Mak \& Coniam, 2008) and retains the probability for collective knowledge advancement and language development 
(Lund, 2008). Wiki technologies enhance effective collaboration in a constructivist approach to language learning through peer interaction and learner-instructor interaction, but are less effective in communicating with peers and co-constructing products (Zorko, 2009) because learners generally communicate less on why they make certain contributions or modification. Wheeler \& Wheeler (2009) concluded that Wiki's collaborative language writing occurred in publicizing students' Wiki language writing tasks. In fact, Wheeler \& Wheeler found students were reluctant to edit each other's writing although overall students reported that their academic writing skills have had improved through their formal participation in the Wiki. This finding was validated by Kessler's study (2009) that students were able to meet the knowledge and skills subcomponents of ability, but lacked the motivation and/or confidence subcomponents of willingness to edit other students' writing; even though they actually contributed to the collaborative task in the form of peer- and self-editing.

Language learning in Wiki environments is more than just cognitive and mechanical interaction via reading, writing, and editing their own contributions and the contributions of others. The aim is to focus on the attitudes of students toward using Wikis as language learning tools. Some critical issues are identified in the literature integrating Wiki instructions and technology to support language learning such as persistent technology challenges (Matthew \& Felvegi, 2009), lack of motivation and confidence subcomponents of willingness to edit the writing of other students (Kessler, 2009), and reluctance to contribute and edit Wiki content (Kessler, 2009; Wheeler $\&$ Wheeler, 2009). These issues thought of as a lack of understanding in the attitudes of students toward Wiki technology and Wiki instructions in language learning. Students' attitude toward to Wiki technology and instructions involve beliefs, feeling, values, and dispositions of social learning. Hazari, North, and Moreland (2009) concluded a need exists to examine Web 2.0 technologies and instructions as they relate to the attitudes of students. Brooks (2008) corroborated Web 2.0, such as Wiki, is an attitude, and not a technology.

The attitude of the HCC students toward Wiki may have some implications for their actual Wiki interactions. This study purposed to examine the predictive relationship between attitude toward Wiki use and Wiki interaction in a single cultural background classroom. It is hypothesized that there is positive relationship between attitudes of learners toward language learning in Wiki learning environments and Wiki interaction in a Chinese ESL classroom.

\section{Method}

\subsection{Study Participants}

The participants were recruited from the first-year English classes at a four-year, public polytechnic university in Taiwan in fall 2009. Each student was required to finish two group Wiki English composition assignments with five other students. Each group assignment was to be finished in six weeks. Participants were encouraged to focus on language accuracy while actively participating and interacting with their peers in various ways when accurate English writing skills are the main goals for this assignment. Two hundred and four freshman students in an undergraduate Management program and Engineering program participated voluntarily by responding to the online survey.

In this convenience sample $(N=204)$, majority of the participants were male $(n=147$, $72.059 \%)$. The age composition was as the follows: (1)< 18 ( $n=13,6.373 \%)$, and (2) 18 $25(n=191,93.627 \%)$. The participants were 
also asked to assess their computer skills. As a result, $23(11.275 \%)$ rated themselves as no experience or little experience, 129 (63.235\%) as novice, $46(22.549 \%)$ as intermediate, and 6 $(2.941 \%)$ as expert.

\subsection{Measurement of Research Variables}

\subsubsection{Predictor variable}

Attitude toward Wiki interaction was measured by 5 online survey items (see Table 1) with a five point Likert scale (1: Strongly Disagree; 2: Disagree; 3: Neutral; 4: Agree; 5: Strongly Agree). These five items developed were based on students' beliefs, feeling, values, and dispositions to Wiki environment for language learning. Each participant's responses to those five survey items were summed up to obtain a total score of attitude toward Wiki, ranging from 5 to 25 . The higher the total score was, the more positive the attitude toward Wiki.

\subsubsection{Criterion variables}

Wiki interaction was measured by 7 online survey items (see Table 1) with a five point Likert scale (1: Strongly Disagree; 2: Disagree; 3: Neutral; 4: Agree; 5: Strongly Agree). Each participant's responses to those seven survey items were summed up to obtain a total score of Wiki interaction, ranging from 7 to 35. The higher the total score was, the greater the Wiki interaction. The value of the Cronbach alpha reliability coefficient estimated from the data collection, $734(N=204)$, was above the preset criterion (.700) and indicated an adequate internal consistency among survey items.

Table 1. Survey Items Measuring Research Variables

\begin{tabular}{|c|c|c|}
\hline Variable & Item & Item content \\
\hline \multicolumn{3}{|c|}{ Attitude toward Wiki } \\
\hline & 1 & I perceive Wiki as highly related to English learning. \\
\hline & 2 & I like to use Wiki to support my English learning. \\
\hline & 3 & I like to use Wiki. \\
\hline & 4 & I perceive the use of Wiki in English learning as convenient. \\
\hline & 5 & I prefer to use paper and pencil to write English composition. \\
\hline \multicolumn{3}{|c|}{ Wiki Interaction } \\
\hline & 1 & I spent a great deal of time to write my English composition. \\
\hline & 2 & I spent a great of time to edit my own English composition. \\
\hline & 3 & I enjoyed reading my teammates' English compositions. \\
\hline & 4 & $\begin{array}{l}\text { I used other references books while editing my teammates' } \\
\text { English compositions. }\end{array}$ \\
\hline & 5 & $\begin{array}{l}\text { By editing my teammates' English composition, I improved } \\
\text { my own English proficiency. }\end{array}$ \\
\hline & 6 & $\begin{array}{l}\text { I spent a great of time to edit my teammates' English } \\
\text { compositions. }\end{array}$ \\
\hline & 7 & $\begin{array}{l}\text { When editing teammates' English compositions, I took time to } \\
\text { think on how to edit them. }\end{array}$ \\
\hline
\end{tabular}




\subsection{Data Analysis}

For the current study, all data analyses were conducted with the SPSS 17.0. A simple regression analysis (Cohen, Cohen, West \& Aiken, 2003) was conducted to assess the predictive relationships between attitude toward Wiki and Wiki interaction. Furthermore, the alpha level was set at the .05 level for the significance test.

\subsubsection{Significance test}

The one-tailed $t$ test of the regression coefficients associated with attitude toward Wiki would determine if there was a statistically nonzero predictive relationship between attitude toward Wiki and Wiki interaction. The null hypothesis for the one-tailed t tests was set up as $H_{0}: \mathrm{b}=0$. The alternative hypothesis was set up as $H_{0}: \mathrm{b}>0$ due to the expectation of a positive predictive relationship between research variables.

\subsubsection{Effect size}

The squared multiple correlation coefficient $\left(R^{2}\right)$ (Cohen et al., 2003) was computed to estimate the proportion of variance in Wiki 2.0 interaction associated with, then predictable by attitude toward Wiki. Moreover, the adjusted squared multiple correlation coefficient (adjusted $R^{2}$ ) was obtained to correct the positive bias of the sample squared multiple correlation coefficients and serve as a more accurate estimator of their population counterparts (Cohen, et al., 2003).

\subsubsection{Checking regression assumptions}

Prior to the $t$ test of the regression coefficient associated with attitude toward Wiki, the assumptions of normality, linearity and homogeneity of variances, and independence were checked for severe violations with various statistical graphs and index (Norusis, 2008). The assumption of normality was evaluated by the histogram and normal Q-Q plot of the studentized residuals. The assumptions of linearity and homogeneity of variances were assessed by the scatterplot of standardized predicted scores and studentized residuals. The assumption of independence was assessed by the Durbin-Watson statistic of residuals.

\section{Results}

\subsection{Descriptive Statistics of Research Variables}

The descriptive statistics of all research variables are listed in Table 2. The Means and Medians for both variables, Attitude toward Wiki and Wiki interaction, are close to each other. The low Standard Deviations for both variables indicated that most of the data points centered around the Means and Medians. This indicated normality assumption on the data distributions which was further examined the histogram and the normal Q-Q plot.

Table 2. Descriptive Statistics of Research Variables $(\mathrm{N}=204)$

\begin{tabular}{lccccc}
\hline Variable & $M$ & $M d n$ & $S D$ & Min & Max \\
\hline Attitude toward Wiki & 16.392 & 16.000 & 2.957 & 5.000 & 25.000 \\
Wiki interaction & 23.382 & 23.000 & 4.432 & 7.000 & 35.000 \\
\hline
\end{tabular}

Note. M: Mean; Mdn: Median; SD: Standard deviation; Min: Lowest score; Max: Highest score. 


\subsection{Assumption Checking in Regression Analysis}

Based on the shape of the histogram (see Figure 1) and the pattern of data points in the normal Q-Q plot (see Figure 2), violation of the normality assumption was not a concern. As to the assumptions of linearity and homogeneity of variances, the pattern of data points in the scatterplot of standardized predicted scores and studentized residuals (see Figure 3) suggested no severe violation of them. The actual DurbinWatson statistic, 1.719, was not sizably different from 2 and indicates no severe violation of the independence assumption. In conclusion, due to no severe violation of those assumptions in regression, the results of the $t$ test should be valid.

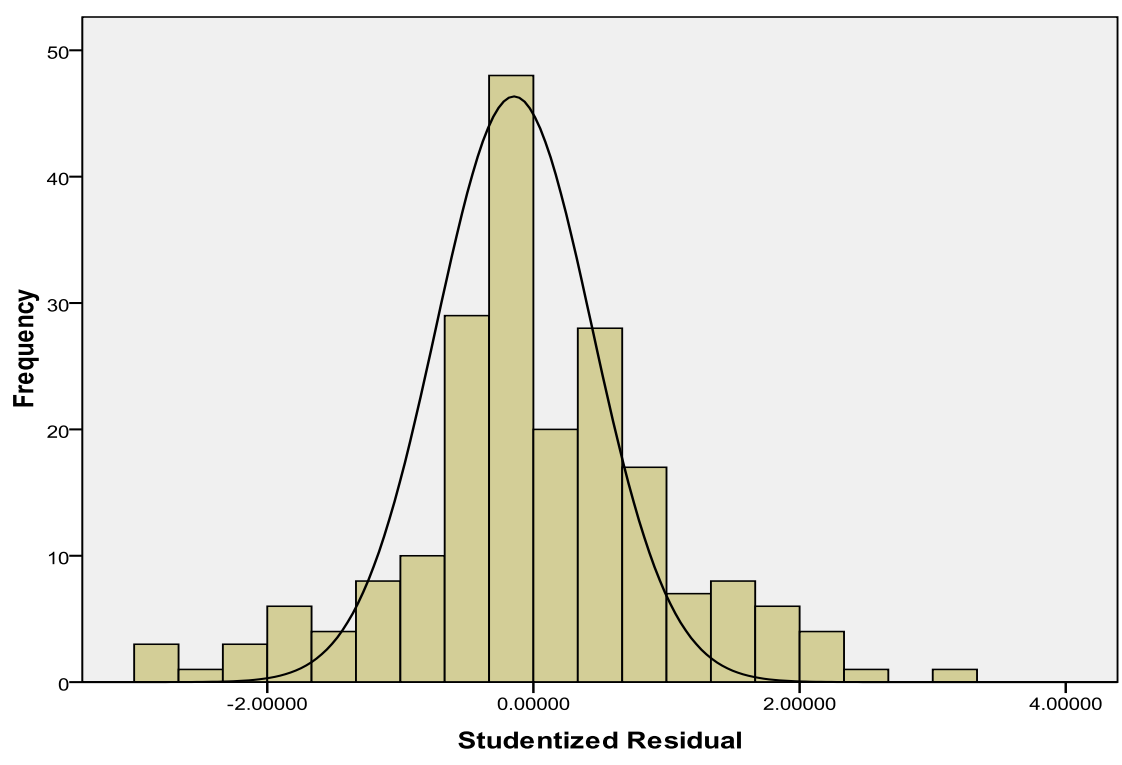

Figure 1. Histogram of Studentized Residuals

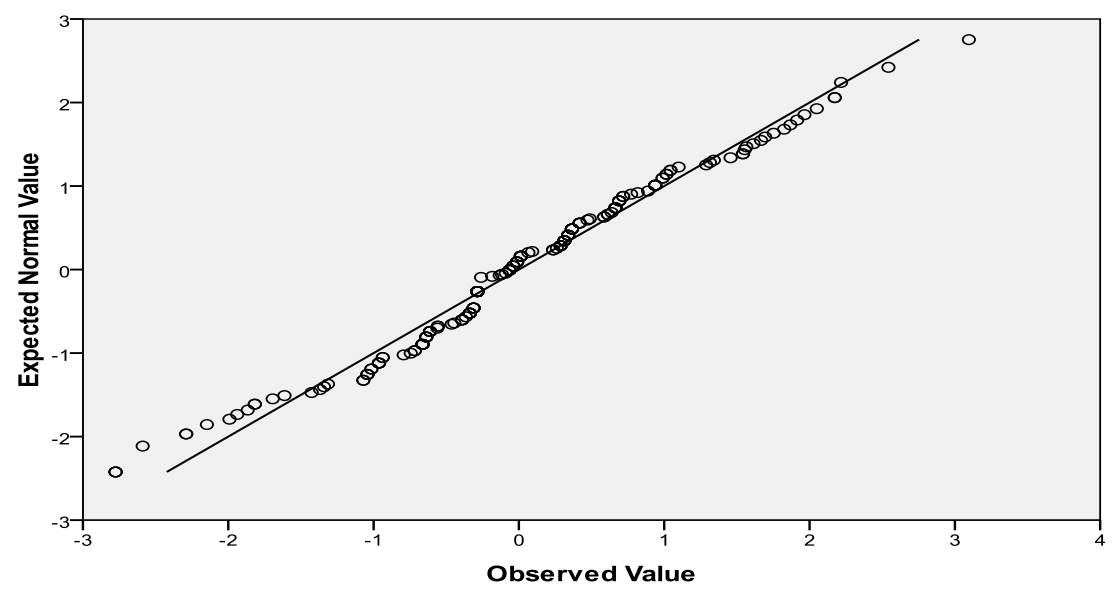

Figure 2. Normal Q-Q Plot of Studentized Residuals 


\section{Dependent Variable: Interaction_Wiki}

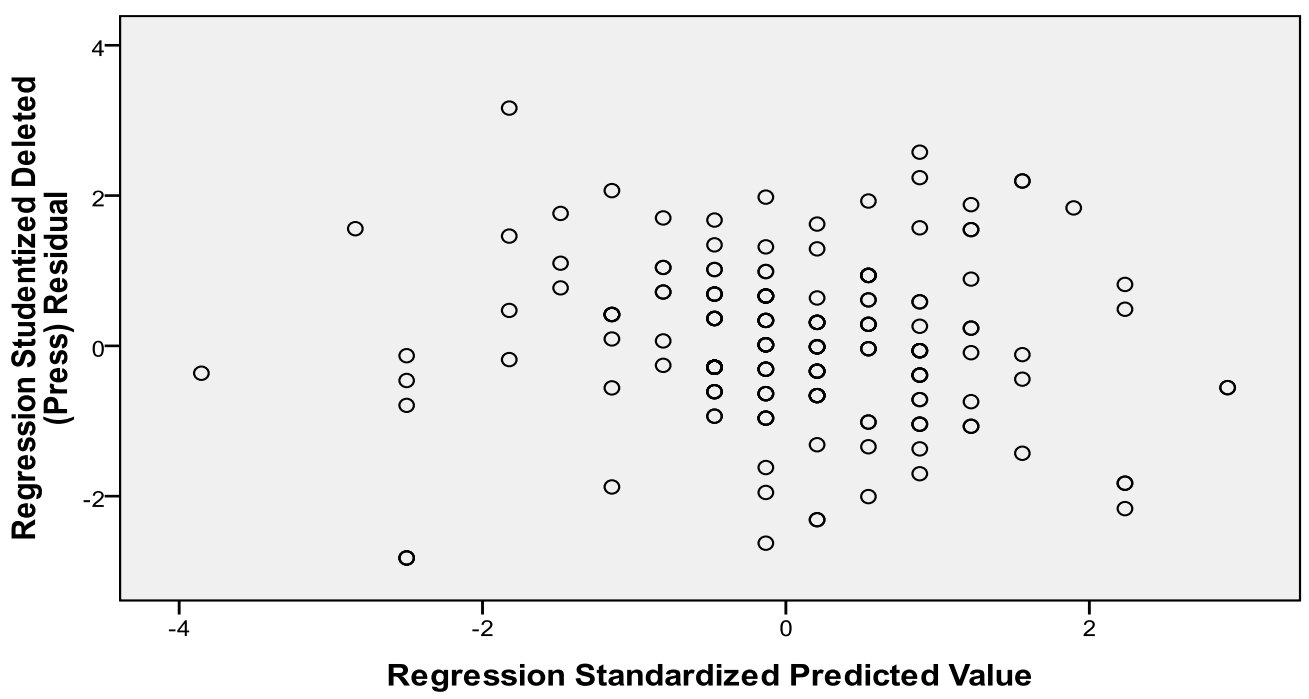

Figure 3. Scatterplot of Standardized Predicted Scores and Studentized Residuals

\subsection{Simple Regression Model with Attitude toward Wiki as the Predictor Variable}

The results of the $t$ test for the regression coefficient associated with attitude toward Wiki supported a nonzero, positive predictive relationships between attitude toward Wiki and Wiki interaction, $t(202)=14.761, p<.05$. The values of the $R^{2}, .519$, and the adjusted $R^{2}$, .517 , further supported the conclusion from the $t$ test.

In conclusion, there was a medium to strong, positive relationship between attitude toward Wiki and Wiki interaction, so that attitude toward Wiki could serve as a useful predictor for Wiki interaction.

\section{Discussion}

The results of this study suggested a medium to strong, positive relationship between attitude toward Wiki and Wiki interaction in a Chinese
ESL classroom. To support social interaction of Chinese ESL students in Wiki (writing, reading and editing their own and others' writings), it may be beneficial to understand, to improve, and to shape the attitudes of these students toward Wiki ESL learning by helping them understand what, how, and why collaborative Wiki writing may support their learning of English.

In this study, there were five online survey items to measure students' attitude toward in using Wiki as learning tools while Wiki interactions, evaluated by seven online survey items, were measures of students' experiences in engaging in reading, writing, and editing their own and others' English compositions. Three dimensions of Constructs for Web 2.0 Learning Environments ( $\mathrm{Tu}$, Blocher \& Roberts, 2008) were aligned to this study to shed light on understanding the attitudes of students toward the use of Wiki in language learning and Wiki interactions (Cognitive, Social, and Interaction Dimensions). 
The cognitive dimension concerns how learners think about what, how and with whom they will contribute their contents through interactions. The social dimension concerns the individual and the social contexts which constitute the relationships between individual, social, and cultural environments. The integration dimension concerns how learning is situated in social and collaborative activities to build communities.

Based on these three dimensions for Web 2.0 Learning Environments, four factors seem accentuate positive attitudes of students toward Wiki: (1) familiarity with the Wiki process, (2) understanding of the value of Wiki in ESL, (3) positive social relationships, and (4) sufficient time to build a Wiki community.

\subsection{Familiarity with Wiki Process}

Not all students are familiar with Wiki technology. One cannot assume all younger learners are familiar with Wiki technology even though younger generations are confident with social media for personal entertainment applications. In this study, $94 \%$ of students between 18-25 is considered to be of the E-Generation. However, only $26 \%$ of students judged their computer skills to be Intermediate (23\%) and Expert (3\%) levels; and only 10\% of them had experienced Wiki technology. Therefore, integrated Wiki-based exercises are required to familiarize students with Wiki technologies. Wiki interactions engage learners in reading, writing, and editing their own English compositions and those of others. HCC students who are not familiar with Wiki technology are less likely to request assistance from instructors, preferring to work on their own or seek support from classmates in their inner circle (Bond, 1991). Wiki-based exercises and Wiki self-introduction implemented prior to the use of Wiki will provide an opportunity for students to build familiarity with the technology. These Wiki warm-up exercises should be informal and not graded to reduce learning anxiety. These warm-up exercises also would increase learners' positive online learning and Wiki technology experiences. This would fulfill what Lambert, Kalyuga, and Capan's (2009) suggested that effective Web 2.0 learning environments require students to have prior distance learning and technology experiences.

\subsection{Understanding of the Value of Wiki in ESL Instruction}

It is critical to explain to students the rationale behind integrating Wiki technologies into the learning experiences. Students may have substantial computer experience; however, this does not equate to a good understanding of technology, Wiki technology, and Wiki technology in language learning values. In this study, more than three-fourths of the students (79\%) had used the computer over three years, while $72 \%$ of them use computers 1-6 hours daily. With these high usages and extensive computer experiences, only $10 \%$ of students had experienced Wiki technology. The HCC students require deeper and richer social contexts to build their social relationships with others in a learning community. Therefore, they need to have a better understanding of how and why Wiki can support their learning.

The benefits Wiki provides for language learning should be conversed with learners before actual Wiki language learning activities occur: content creations, revisions, and collaboration versus cooperation. The most critical adjustment in attitudes toward Wiki language learning require that instructors inform learners, particularly HCC learners, that editing the writing of others and revisions are not corrections, antagonistic, offensive, or impolite. Rather it is a collaborative form of writing and learning within a positive learning 
community. It is not competition, but simply helping and supporting each other. Because of the high deference to authority, instructors need to constantly remind and reinforce this concept and understanding to HCC students. This finding is similar to what Kessler's (2009) and Wheeler and Wheeler's (2009) studies reveal in that learners are unwilling to edit the writing of other learners. Additionally, Hollingshead \& McGrath (1995) argued competitive performance tasks do not support the context of social-cultural learning. Therefore, benefits of integrating Wiki to support collaborative language learning should be communicated effectively with learners prior the Wiki activities.

\subsection{Positive Social Relationships}

The HCC students need positive and trusting social relationships to initiate and sustain their learning communities. Instructors should emphasize that effective Wiki language learning should focus on Wiki language creation and editing in additional to building positive relationships. Positive relationships are built through effective communication. Culturally, the HCC students are not encouraged to express themselves. Online profiles have become a means of identity presentation to build social networks (Boyd \& Heer, 2006). The students should be encouraged and perhaps required to create profiles, upload pictures, and create team names while they are creating the Wiki accounts. These activities can be the means to building positive social relationships in their Wiki teams.

\subsection{Sufficient Time to Build the Wiki Community}

HCC students generally require more time to build and foster their own collaborative learning community. They tend to be more cautious during initial interactions, make assumptions based upon the cultural background of a stranger, and ask more questions about a stranger's background (Gudykunst, 1983). Activities designed to engage students in creating and editing group work normally lack non-verbal and social cues. Accordingly, it will take longer for the HCC students to obtain and process enough information about the perspective group member before they can effectively participate. Instructors must allow groups time to build positive and healthy communities. Any instruction regarding the use of tools should provide ample time to allow students to participate. As students become better acquainted with their classmates, they will incorporate them into their inner circle (Bond, 1991). As a result, as time progresses, the HCC students will become more comfortable and have more trust in their classmates. Frequent changes in group membership may cause frustration for students and result in ineffective community learning. In this study, students were given ample time to build a community and to complete community tasks. Students were allowed the first two weeks of instruction to become familiar with Wiki and the Wiki activities. Students had six weeks to complete their team tasks for each of two Wiki assignments. The students stayed with the same teams for the semester. It is believed the Wiki instructional design with ample time may contribute to a positive attitude of students toward Wiki technology in language learning.

\section{Conclusions}

While integrating social networking technologies support language learning, educators frequently focus on the Wiki's potential learning supremacy in cognitive and mechanical interaction in reading, creating, and editing the Wiki content supplied by the student and Wiki content supplied by others. On the surface, learners achieve better learning outcomes in language learning 
through collaborative writing instructions. The relevant literature supported this claim and observed that students, in fact, were reluctant and less motivated to write, and edit the collaborative writings even if perceived learning and actual learning knowledge and skills improved. Wiki is considered "social media." Social aspects should be accentuated to maximize social interaction in Wiki activities by understanding and examining students' attitudes toward language learning in Wiki environments. Wiki's learning power resides in its social aspect which distinguishes it from previous digital media. If one integrates Wiki technology with previous digital media attitude in mind, Wiki technology would simply be just another piece of technology rather than an innovative learning tool. Wiki technology is not just a tool, rather it is an attitude.

This study concludes a medium to strong, positive relationship between students' attitude toward Wiki in language learning and Wiki interaction. Therefore, the attitudes of High-Context Culture students toward ESL Wiki can serve as an effective predictor of Wiki interaction (e.g., creating, self-editing, and editing others' writings). Educators must understand the cultural backgrounds of their students before positive attitudes toward Wiki can be cultivated. One should not assume that all learners from different cultural backgrounds will have the same attitudes toward Wiki instructions. Wiki interactions are deeply rooted in socio-cultural learning psychology. Taking students' cultural backgrounds into account prior to the introduction of Wiki technology into the class is critical. Effective strategies are suggested to improve the attitudes of students to enhance and to maximize language learning in Wiki learning environments. These include familiarizing oneself with the Wiki process, understanding of the value of Wiki in ESL, facilitating positive social relationships, and giving sufficient time to build a Wiki community. Future research should continue examining the process of Wiki instructions for online collaboration from social and cultural aspects. Additionally, multi-cultural learning environments should be examined differently because learners from different cultures may interact differently in a single culture learning environments from multi-cultural environments.

\section{References}

Bond, M. H. (1991). Beyond the Chinese face: Insights from psychology. Hong Kong: Oxford.

Boyd, d., \& Heer, J. (2006). Profiles as conversation: Networked identity performance on Friendster. Paper presented at the Hawai'i International Conference on System Sciences (HICSS-39). Kauai, HI: IEEE Computer Society. Retrieved April 27, 2009, from http://www.danah.org/ papers/HICSS2006.pdf

Braun, S., \& Schmidt, A. (2000). Sociallyaware informal learning support: Potentials and challenges of the social dimension. In Proceedings of the European Conference on Technology-Enhanced Learning (EC-TEL 06). Heraklion, October 2006. Retrieved December 11, 2008, from http:// publications.professional-learning.eu/ Schmidt_Braun_LOKMOL06_final.pdf

Brooks, L. K. (2008). "Old School" meet school library 2.0: Bump your media program into an innovative model for teaching and learning. Library Media Connection, 26(7), 14-16.

Cohen, J., Cohen, P., West, S. G., \& Aiken, L. S. (2003). Applied multiple regression/ correlation analysis for the behavioral sciences ( $3^{\text {rd }}$ ed.). Mahwah, NJ: Lawrence Erlbaum. 
Elgort, I., Smith, A., \& Toland, J. (195). Is Wiki an effective platform for group course work? Australasian Society for Computers in Learning in Tertiary Education, (24), 2.

Farabaugh, R. (2007). "The isle is full of noises": Using Wiki software to establish a discourse community in a Shakespeare classroom. Language Awareness, 16(1), 41-56.

Greenhow, C., Robelia, B., \& Hughes, J. E. (2009). Learning, teaching, and scholarship in a digital age: Web 2.0 and classroom research: What path should we take now? Educational Researcher, 38, 246-259.

Gudykunst, W. B. (1983). Uncertainty reduction and predictability of behavior in low- and high-context cultures: An exploratory study. Communication Quarterly, 31(1), 49-55.

Hazari, S., North, A., \& Moreland, D. (2009). Investigating pedagogical value of Wiki technology. Journal of Information Systems Education, 20(2), 187-198.

Hollingshead, A. B. \&, McGrath, J. E. (1995). Computer-assisted groups: A critical review of the empirical research, in R. A. Guzzo and E. Salas (Eds.), Team effectiveness and decision making in organizations: San Francisco, CA, Jossey-Bass.

Kessler, G. (2009). Student-initiated attention to form in Wiki-based collaborative writing. Language Learning \& Technology, 13(1), 79-95.

Lambert, J., Kalyuga, S., \& Capan, L.A. (2009). Student perceptions and cognitive Load: What can they tell us about E-Learning Web 2.0 course design? E-Learning, 6(2), 150-163.

Lund, A., \& Rasmussen, I. (2008). The right tool for the wrong task? Match and mismatch between first and second stimulus in double stimulation. International Journal of Computer-Supported Collaborative Learning, 3(4), 387-412.

Mak, B., \& Coniam, D. (2008). Using Wikis to enhance and develop writing skills among secondary school students in Hong Kong. System: An International Journal of Educational Technology and Applied Linguistics, 36(3), 437-455.

Matthew, K., \& Felvegi, E. (2009). Learning course content by creating a Wiki. TechTrends: Linking Research and Practice to Improve Learning, 53(3), 67-73.

Norusis, M. J. (2008). SPSS 17.0 statistical procedures companion. Upper Saddle River, NJ: Prentice Hall.

Sue, D. W., \& Sue, D. (1999). Counseling the culturally different: Theory and practice (3rd ed.). Hoboken, NJ: John Wiley \& Sons Inc.

Thompson, C., \& Pennycook, A. (2008). A question of dialogues: authorship, authority, plagiarism. Education Canada, 48(3), 2023.

Tu, C., Blocher, M., \& Roberts, G. (2008). Constructs for Web 2.0 learning environments: A theatrical metaphor. Educational Media International, 45(3), 253-268.

Wheeler, S., \& Wheeler, D. (2009). Using Wikis to promote quality learning in teacher training. Learning, Media and Technology, 34(1), 1-10.

Zorko, V. (2009). Factors affecting the way students collaborate in a Wiki for English language learning. Australasian Society for Computers in Learning in Tertiary Education, 25(5), 645-665. 


\section{Contact the Author}

\section{Hung-Ling Twu, Ph.D.}

National Chin-Yi University of Technology,

Taiwan

E-mail: Hyperconnect@Hotmail.com 